\title{
Complete Dental Management of an Autism and Intellectual Disability Patient under General Anaesthesia: A Case Report
}

\author{
Karin Nadia Firsty, DDS, ${ }^{1}$ Nailur Rahmy Wahdany, DDS, ${ }^{1}$ Dian Lupita Sari, DDS, ${ }^{1}$ \\ Yesri Sasmita Purba, DDS, ${ }^{1}$ Tania Saskianti, DDS, $\mathrm{PhD}^{1}$ and Andra Rizqiawan, DDS, $\mathrm{PhD}^{2}$ \\ ${ }^{1}$ Pediatric Dentistry Department, Faculty of Dental Medicine, Universitas Airlangga, Surabaya, Indonesia \\ ${ }^{2}$ Oral and Maxillofacial Surgery Department, Faculty of Dental Medicine, Universitas Airlangga, Surabaya, Indonesia
}

\begin{abstract}
Autism Spectrum Disorder (ASD) is a neurodevelopmental disorder characterized by persistent deficits in social communication and the presence of restricted interests and repetitive behaviours. Comorbidities following ASD, such as seizure, intellectual disability, and sensory impairment worsen patients' ability to care for themselves. We present the case of a 22-year-old man with autism, intellectual disability and visual impairment who had recurrent pain in his upper and lower left posterior teeth that had cavities. On the first visit, the patient was observed and had panoramic x-ray. Clinical examination could not be done properly due to lack of patient cooperation. Restoration, pulp capping, tooth extraction, and odontectomy were planned under general anaesthesia.
\end{abstract}

Key Words: autism, dental treatment, general anaesthesia, intellectual disability

\section{INTRODUCTION}

Autism spectrum disorder (ASD) is defined as a neurodevelopmental disorder characterized by persistent deficits in social communication and the presence of restricted interests and repetitive behaviours. ${ }^{1}$ Patients with ASD have difficulties in social and emotional reciprocity, ability to start and maintain relationships, use of non-verbal communication, and related to hyper and/or hypo sensorial sensitivity; with severity ranging from mild to severe..$^{2-4}$ The National Institute of Child Health and Human Development described ASD as a a lifelong condition that affects 1 out of every 54 children. The World Health Organization (WHO) estimated that the prevalence of ASD internationally is $0.7 \%$; however, this estimation only covered about $16 \%$ of the global children population. Men are more commonly affected with the ratio of male-to-female closer to $3: 1 .^{1}$

ASD could occur alone or can co-occur other chronic disorders at the same time or in tandem. When it comes with comorbidities, it is associated with worse outcomes and increased health needs. Comorbidities of ASD commonly include seizure/ epilepsy, GI disorders, and psychiatric/ behavioural complaints, including intellectual disability (ID) (IQ<70). ${ }^{3}$ People who are diagnosed with ASD and ID as a comorbidity showed greater reduction in functional independence and adaptive skills. The genes that are associated with ASD are often the same genes associated with ID, which cause an overlap of these two disorders. Individuals with ID are likely associated with physical disabilities such

Corresponding author: Tania Saskianti, DDS, PhD

Pediatric Dentistry Department

Faculty of Dental Medicine

Universitas Airlangga

Surabaya, Indonesia

Email: tania-s@fkg.unair.ac.id 

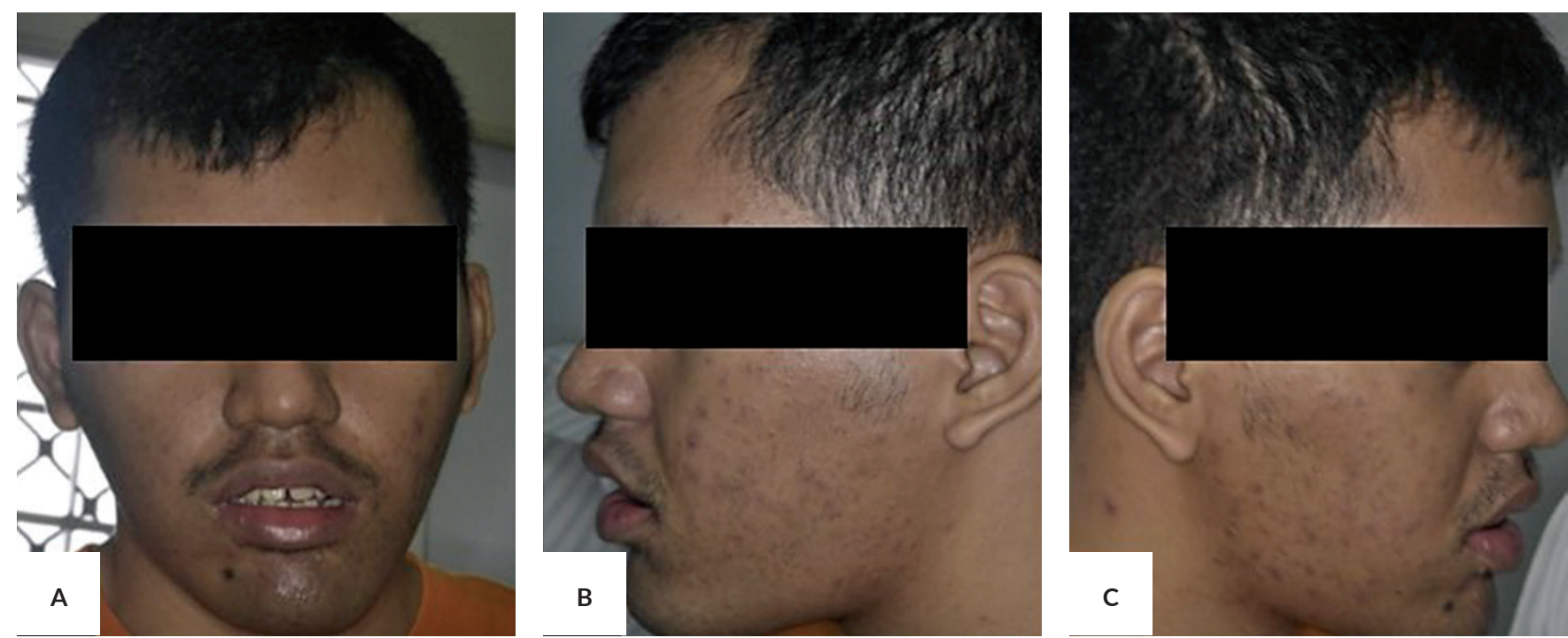

Figure 1. Extraoral profile of the 22-year-old male patient shows mesocephalic facial types with incompetent lips; (A) frontal view; (B) left profile view; (C) right profile view.

as sensory impairments that affect vision and hearing. These conditions are usually seen in people diagnosed with ASD and ID. In addition, conditions of IQ bellow 70 usually persist into adulthood. ${ }^{5}$

People with ASD sometimes experience a higher level of dental anxiety compared to the general population. People with ASD have higher risk of caries since they have difficulty in maintaining oral hygiene, especially in their primary teeth period. This condition worsens with a dietary pattern with increased intake of soft and sweetened food, and gluten-free/ casein-free food that increase the carbohydrate content of saliva. Aside from caries, people with ASD may also have major risk of periodontal lesions and oral trauma. ${ }^{4}$ Treating patients with ASD in a dental office is a challenge for dentists. ${ }^{6}$ Resistance to change in routine makes it difficult for people with ASD to accept a significant change in their daily routine. These conditions combined with having to face new surroundings and staff, possibility of long noisy journeys and uncomfortable waiting areas, can make the whole experience intimidating. ${ }^{7}$ Therefore, in some cases of ASD, dental management under general anaesthesia is necessary, if nonpharmacological method failed. ${ }^{2}$ One indication for the use of general anaesthesia issued by American Academy of Paediatric Dentists (AAPD) is for patients who are unable to cooperate due to lack of psychological or emotional maturity and/or mental, physical, or medical disability. ${ }^{8}$

We presented the case of patient with ASD and ID who underwent complete dental treatment under general anaesthesia.

\section{CASE REPORT}

A 22-year-old man accompanied by his parents consulted at the Universitas Airlangga Dental Hospital

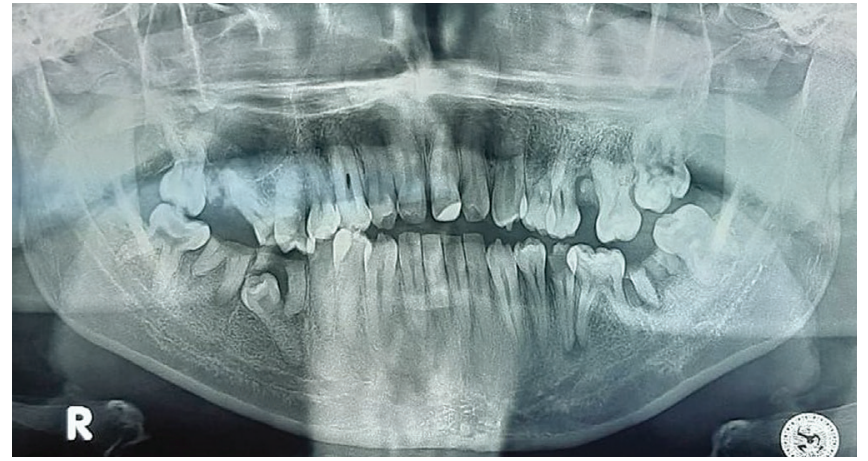

Figure 2. Panoramic radiograph shows permanent dentition with several teeth remnant and radiopaque area surrounding them. There are some misaligned teeth and impacted teeth due to tooth remnant.

with a major complaint of recurrent pain in his upper and lower left posterior teeth. The patient was also diagnosed with autism and intellectual disability at 2.5 years of age (Figure 1). His paternal uncle also had intellectual disability. He underwent two surgeries under general anaesthesia due to visual impairment (cataract and glaucoma) at 6 and 8 months of age. Mild seizure was detected at 2 years of age and he completed 4 years of therapy. Several drugs that were prescribed, such as sertraline $50 \mathrm{mg}$, ariprirazole 10 $\mathrm{mg}$, and potassium chloride, should be stopped a day before surgery. Medical history of hypertension, diabetes mellitus, asthma, and allergy were denied by the parents.

On the first visit, the patient was observed and underwent complete blood count examination, panoramic and thoracal $\mathrm{x}$-ray (Figure 2). Clinical intra-oral examination could not be done due to lack of patient cooperation. First assessment from the panoramic radiographic was multiple pulpitis, 
gangrene radix, and impacted tooth. General anaesthesia was chosen for the purpose of behaviour management. The patient was then referred to a neurologist and psychiatrist for a pre-surgery consultation. In the next five days, the patient was referred back to dental hospital with no contraindication to perform general anaesthesia. The parents were well informed about the method, process, and side effects of the dental and general anaesthesia procedure, and agreed to continue the treatment. A consent form was signed prior to general anaesthesia procedure.

\section{CASE MANAGEMENT}

General anaesthesia was chosen for the purpose of behaviour management due to lack of patient cooperation. The surgery was scheduled 12 days after his second visit. He was hospitalized a day before surgery was performed. Intraoral examination was not properly conducted. Prophylactic antibiotic, cefazolin $2 \mathrm{~g}$ drip in $\mathrm{NaCl}$ 0.9\% 100 $\mathrm{ml}$, was given one hour before the procedure. Omeprazole $40 \mathrm{mg}$, ondansetron $8 \mathrm{mg}$, and midazolam $2 \mathrm{mg}$ were given for premedication. Intravenous sedative and analgetic were administered and the condition was maintained using sevofluran inhalation. There were no spontaneous respiration and movements during the procedure.

Full intraoral examination was performed after the patient had been placed under general anaesthesia in the operating theatre (Figure 3). He was diagnosed to have pulpitis, reversible, on 18,24, 25, 27,38, 44, and 45; persistence of residual roots and necrosed pulp on 16, 17, 35, 37, 46, and 47; and 48 paramolar tooth impaction.

Several treatments were performed: preventive resin restorations (PRR) on 18, 24, 25, and 44; composite restorations on $27,38,45$; teeth extractions on $16,17,35,37$, 46, and 47; and odontectomy on 48 (Figures 4-6).

PRR using resin-based composite was choosen due to its minimal exploratory openings in enamel and isolated carious lesions were removed without any extension into the surrounding healthy tooth. ${ }^{9} \mathrm{PRR}$ protects the tooth by sealing the deep grooves and creating a smooth, easy-toclean surface. This method will protect the teeth from decay for a long time. ${ }^{10}$

Composite resin was chosen for direct permanent restorative material due to its longevity, biocompatibility,
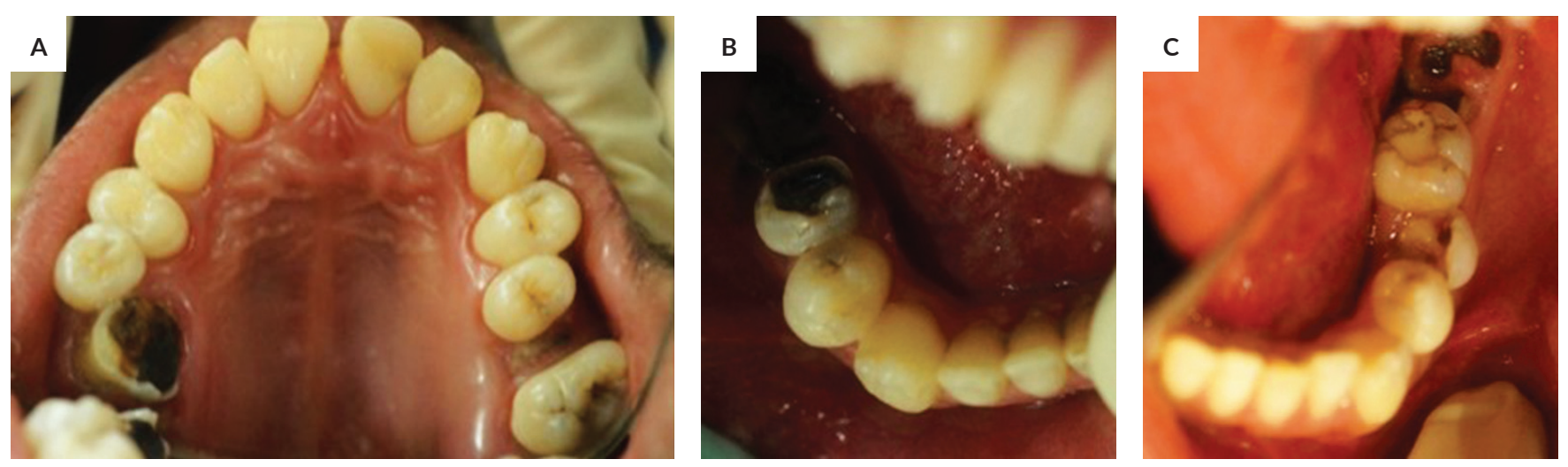

Figure 3. Intraoral profile of the patient pre-operative shows blackened-cavities in some premolars and molars teeth (A) maxillary profile (B) mandible dextra profile; (C) mandible sinistra profile.
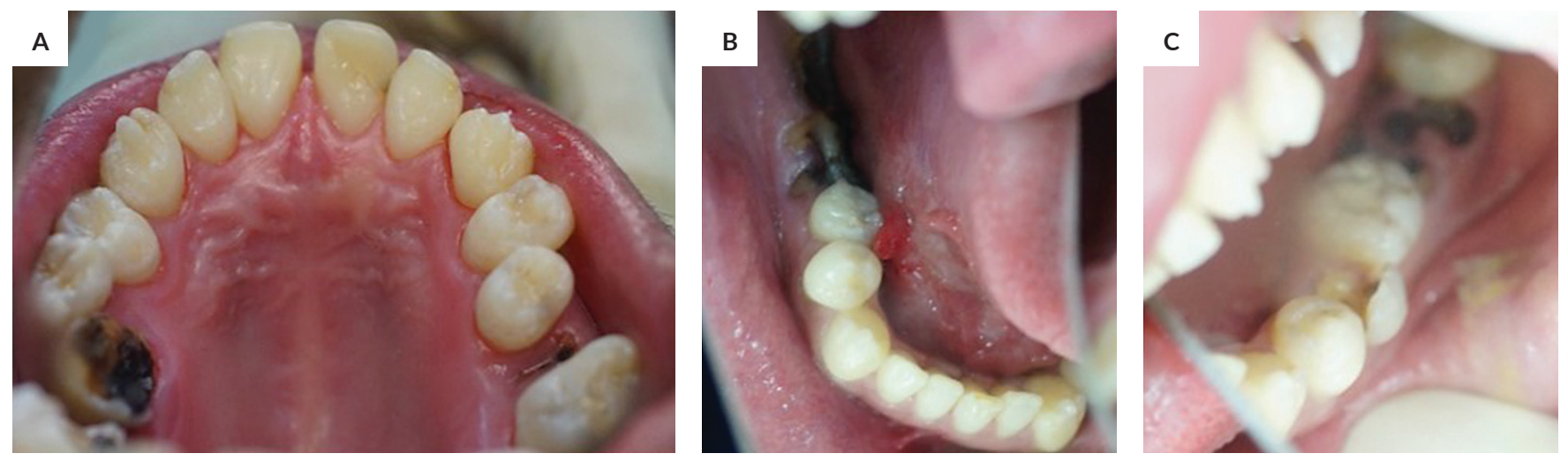

Figure 4. Intraoral profile post tooth restoration, preventive resin restoration on teeth $18,24,25$, and 44 ; composite restoration on teeth $27,38,45$ (A) maxillary profile (B) mandible dextra profile; (C) mandible sinistra profile. 

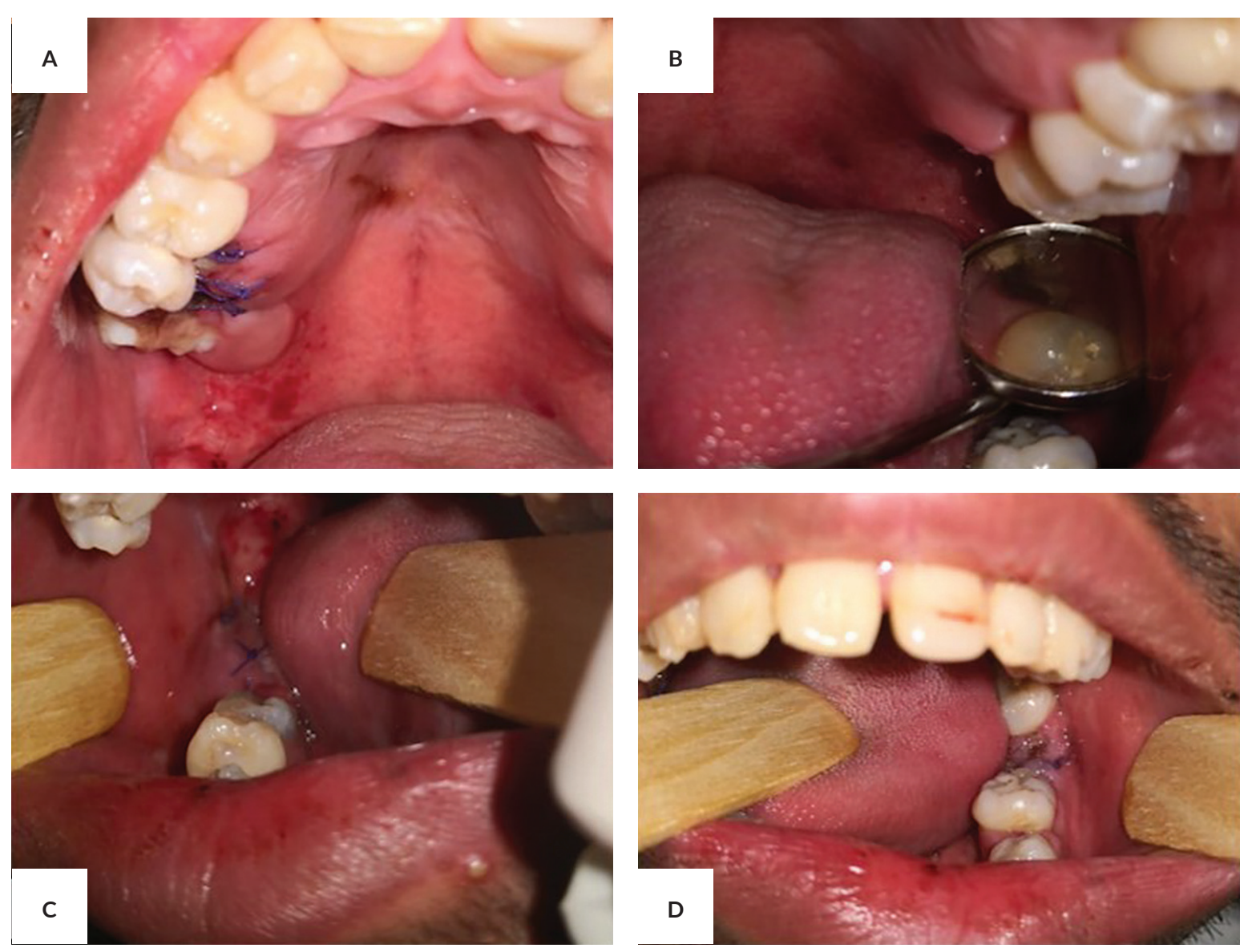

Figure 5. Post-operative intraoral after surgical procedure (A) tooth extractions on 16, 17; (B) restoration on 24, 25, 27; (C) tooth extraction of 46, and 47 and odontectomy 48; and (D) tooth extraction of 35, 37.

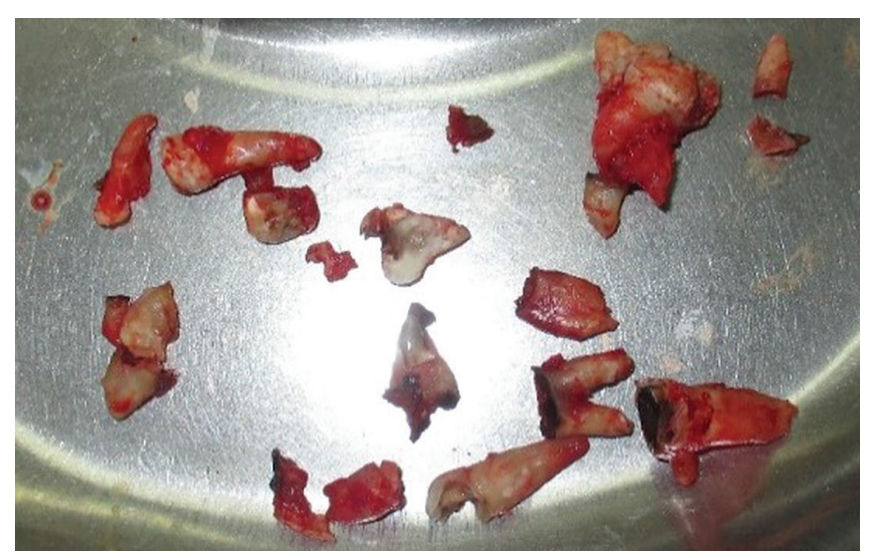

Figure 6. Extracted residual teeth.

marginal leakage and predisposal secondary decay, and better aesthetic than other material. ${ }^{11}$ Extractions were chosen due to severe dental decay cannot be treated.

The patient regained consciousness six hours after induction. There were no complications. Vital signs after procedure showed blood pressure of $120 / 70 \mathrm{mmHg}$, pulse rate of $85 /$ minute, oxygen saturation of $98 \%$, respiratory rate of $18 /$ minute, and temperature of $36^{\circ} \mathrm{C}$. Post-surgery instructions were given to the parents: maintain oral hygiene, drink a little amount of water and slowly, soft and warm diet was allowed if there were no nausea and vomiting. Several drugs were given intravenously: omeprazole $40 \mathrm{mg}$ and ondansentron $8 \mathrm{mg}$ (each was given every 12 hours for 1 day) and ketorolac $30 \mathrm{mg}$ was given every 8 hours for 2 days.

The following day, the patient's intra oral condition was examined. The restorations remained intact. The wounds from multiple extractions were properly covered by silk suture. The parents stated that there was no complaint from or any discomfort in the patient. Patient was discharged the day after surgery and scheduled to have the dental check-up one week later.

\section{DISCUSSION}

Autism is known as a spectrum neurodevelopmental disorder that appears in early childhood, within the first 3 years of life. Autism is defined as an incurable disease and may continue into adulthood. Patient with autistic spectrum disorders typically shows difficulties in communication and social interactions. ASD is also characterized with restricted 
and repetitive interests and activities. Symptoms of ASD that appear in infants include social deficits of interaction, failure to respond to name, lack of pointing fingers and eye contact, delayed speech. Young children with ASD tend to isolate themselves from other children. They also present with inability to participate in a group, empathize, and join social conversations. In adulthood, there is still persistence of social and communication deficits, even though some improvements may be seen. However, some studies showed that more than half of adult ASD patients have language below the level of a ten-year-old. The repetitive behaviour persists in $90 \%$ of adult patients although some uncommon concerns and complex stereotypes may decrease. Autism is the most severe developmental disability; it might be associated with intellectual disability. Intellectual disability is characterized by significant limitations of intellectual function and adaptive behaviour. The degree of intellectual disability varies from mild to profound. Autism is usually followed by intellectual disability with a degree of severity severe (IQ below 50) to profound (IQ below 25). It co-occurs with intellectual disability in $38-75 \% .^{12,13}$ Autism with low IQ also has a strong association with epilepsy due to worse neurocognitive and interneural dysfunction. One third of children with autism also reported experiencing epilepsy., ${ }^{2,714}$

ASD with and without intellectual disability tends to have behavioural problems such as inattention/hyperactivity, anxiety, depression, self-injury, aggression toward others, sensory problems, excessive fearfulness, and temper tantrums. Similar to children, ASD in adulthood that co-occurs with intellectual disability is likely to have higher risk of depression and anxiety than without intellectual disability. ${ }^{13}$

Proper intervention is needed to achieve successful dental treatment. Behaviour management should be done and varies for each individual. Desensitization is the first thing to do at the first visit. The U.S. Department of Health and Human Services suggests desensitization, to help the patient become familiar with the office and the dental team, positive reinforcement and use of immobilization techniques. If all other strategies fail, they recommend to use pharmacological options or general anaesthesia. The tell-show-do technique could not be done due to difficulties in overcoming the patient's fears. This technique may be successful in patients with ASD without intellectual disability. Children can be restrained if desensitization fails, but not adults. Either inhalation or intravenous sedation can be done if physical and mechanical restraint fails, but should meet some requirements, depending on patient condition. General anaesthesia for dental management of differently abled patients is indicated for: intellectual disability, multiple and complex extractions, teeth that require surgical removal and suturing, and symptomatic teeth in more than a quadrant. ${ }^{3,12}$

Surgical antibiotic prophylaxis was given to reduce postoperative infection of the surgical site. We also considered the patient's special condition. In an ASD patient, cefazolin was well known to significantly reduce the patient's repetitive behaviour aside from its antibacterial effect. ${ }^{15}$ Before the procedure begins, the patient's behaviour needs to be taken care of. Knowing that the patient might not be communicative and to minimize disruption, the environment should be made less noisy and waiting time should not be prolonged. Physical restraint should be avoided; but in some cases, such as this case, physical restraint was conducted in order to control the situation effectively. ${ }^{16}$

The use of intravenous propofol is also effective in anaesthesia for patients with special needs due to developmental delay, anxiety, and behavioural disorder. It also reduces postoperative nausea and vomiting (PONV). ${ }^{17}$ Patients with special needs have difficulties in managing post-surgical pain; therefore, we continuously monitored his post-surgical medication. After the procedure, omeprazole and ondansetron were also given to reduce postoperative nausea and vomiting (PONV) and gastroesophageal reflux. ${ }^{16}$

In this case, patient regularly visited a paediatric dentist and underwent restorative treatment during his childhood. According to the parents, the patient was cooperative during his previous treatment in mixed dentition phase. His old restorations of central incisive and first permanent molar were well preserved. As the patient grew older, the dentist found it difficult to handle the patient for clinic- based treatment; thus, he was referred to a dental hospital for further treatment. Behaviour shaping for disabled patients should be done regularly, and the treatment should be done promptly and efficiently. ${ }^{12,13}$

Parents' and caregivers' perceptions about the need for dental treatment for children and adults with special care needs, especially ASD and intellectual disability in this case, depends on various factors. Their perceptions are determined by stress, from the social attitudes of others, as well as financial, employment, and time-related burdens. Therefore, dental teams may need an individualized and structured approach. It is important to provide stability and familiarity. It should suit the individual's characteristics, involve the patient's family, and create a routine that can be followed every dental visit. $8,9,18$

Although patients with ASD and intellectual disability usually lack self-determination, they can be trained. A previous study showed a significant lower level of autonomy, psychological empowerment and self-realization in patients with ASD and intellectual disability. ${ }^{19}$ The caregivers/parents of this patient were cooperative and willing to be informed and educated about the process, risks, and prognosis. They also had no socio-economic issue for the patient to undergo dental treatment under general anaesthesia. Good communication and cooperation between the patient, parents, and dentist play an important role in successful treatment. Current evidence suggests that dental treatment under general anaesthesia in individuals with ASD are performed commonly and may be necessary for quality dental care in patients with ASD. Furthermore, periodic visits are needed to maintain the oral health of the patient. ${ }^{20,21}$ 


\section{CONCLUSION}

When performing dental treatment and general anaesthesia for ASD patient with intellectual disability, patient's safety and benefit should be considered. The number of teeth that needs to be treated, difficulty and severity of the case, and difficulty in managing patient behaviour are aspects that need to be considered. Complete dental treatment under general anaesthesia was chosen in this case to take care of patient's oral problems in one sitting. For further treatment, behavioural shaping can be done to reduce patient's anxiety and obtain good post-operative attitude.

\section{Statement of Authorship}

All authors participated in the data collection and analysis and approved the final version submitted.

\section{Author Disclosure}

All authors declared no conflicts of interest.

\section{Funding Source}

This research was fully funded by Community Service Grant from Faculty of Dental Medicine Universitas Airlangga 2019.

\section{REFERENCES}

1. Hodges, H., Fealko, C. and Soares, N. Autism spectrum disorder: definition, epidemiology, causes, and clinical evaluation. Transl Pediatr. 2020: 9(S1);S55-S65. doi: 10.21037/tp.2019.09.09.

2. Czornobay LFM, Munhoz EA, Lisboa ML, Rath IB, de Camargo A. Autism spectrum disorder: review of literature and dental management. World J Stomato. 2018 November 26; 6(2): 6-18.

3. Casanova MF, Frye RE, Gillberg C, Casanova EL. Editorial: Comorbidity and Autism Spectrum Disorder. Front Psychiatry. 2020 Nov 20; 11:617395. doi: 10.3389/fpsyt.2020.617395.

4. Ferrazzano, G. F. et al. Autism spectrum disorders and oral health status: Review of the literature', European Journal of Paediatric Dentistry,. 2020; 21(1):9-12. doi: 10.23804/ejpd.2020.21.01.02.

5. Thurm A, Farmer C, Salzman E, Lord C, Bishop S. State of the field: differentiating intellectual disability from autism spectrum disorder. Front Psychiatry. 2019 Jul 30; 10:526. doi: 10.3389/fpsyt.2019.00526.
6. Chandrashekhar S, S Bommangoudar J. Management of autistic patients in dental office: a clinical update. Int J Clin Pediatr Dent. 2018 May-Jun; 11(3):219-27. doi: 10.5005/jp-journals-10005-1515.

7. Nizarali N, Boyle CA. Providing dental care under general anaesthesia for people with autism. Austin Journal of Autism \& Related Disabilities. 2017; 3(2).

8. American Academy of Pediatric Dentistry. Behavior guidance for the pediatric dental patient, The Reference manual of Pediatric Dentistry. 2015. doi: 10.1016/b978-0-323-60826-8.00024-9.

9. Pandiyan NJ, Hedge A. A Clinical comparison on success of sealant and preventive resin restoration on caries prevention. Malaysian Journal of Apllied Sciences. 2016; 1(2):71-7.

10. L. Sri Varsha. Recent advances in preventive resin restoration (PRR). Research J Pharm and Tech. 2019; 12(1):382-4. doi: 10.5958/0974360X.2019.00069.6.

11. Patki B. Direct permanent restoratives - amalgam vs composite. J Evol Med Dent Sci. 2013; 2(46):8912-8.

12. Gupta PV. Pediatric Dentistry for Special Child. 1st ed. New Delhi: Jaypee Brothers. 2016:3-81.

13. Kurzius-Spencer M, Pettygrove S, Christensen D, Pedersen AL, Cunniff $\mathrm{C}$, et al. Behavioral problems in children with autism spectrum disorder with and without co-occurring intellectual disability. Res Autism Spectr Disord. 2018; 56(April):61-71.

14. Anand V, Jauhari P. Autism, Epilepsy and intellectual disability: a clinical conundrum. Indian J Pediatr. 2019; 86(10):877-8.

15. Barnhill K, Mosher D, Mong J, et al. Reduction of ritualistic behavior in a patient with autism spectrum disorder-treated with antibiotics: a case report. J Psychiatry Psychiatric Disord. 2017; 1(3):148-53

16. Short JA, Calder A. Anaesthesia for children with special needs, including autistic spectrum disorder. $\mathrm{Br} \mathrm{J}$ Anaesth. Continuing Education in Anaesthesia, Critical Care \& Pain. 2013; 13(4):107-11.

17. Chan V, Skowno J. A practical approach to propofol-based total intravenous anaesthesia (TIVA) in Children. ATOW. 2018; 392.

18. Wiener RC, Vohra R, Sambamoorthi U, Madhavan SS. Caregiver burdens and preventive dental care for children with autism spectrum disorder, developmental disability and/or mental health conditions: National Survey of CSHCN, 2009-2010. Matern Child Health J. 2016; 20(12):2573-80.

19. Chou YC, Wehmeyer ML, Palmer SB, Lee J. Comparisons of selfdetermination among students with autism, intellectual disability, and learning disabilities: a multivariate analysis. Focus Autism Other Dev Disabl. 2017; 32(2):124-34.

20. U.S. Department of Health and Human Services. Practical Oral Care for People with Autism. National Institute of Dental and Craniofacial Research [Internet] 2009. [cited 15 March 2021]. Available from: https://www.nidcr.nih.gov/sites/default/files/2017-09/practicaloral-care-autism.pdf

21. Widyagarini A, Suharsini M. Dental care for children with autism spectrum disorder. Dent J (Majalah Kedokt Gigi). 2018; 50(3):160. 